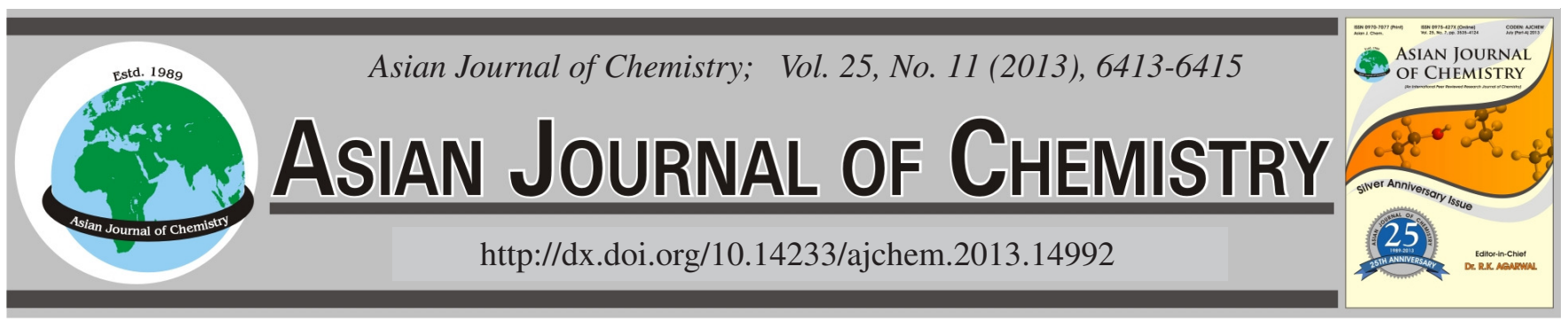

\title{
Preparation of ZnO Microtube Capsule for Drug Delivery System via Microemulsion-Sol-Hydrothermal Method
}

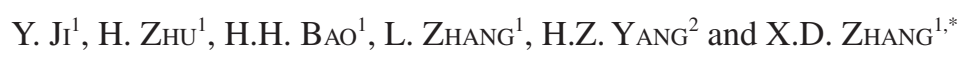

${ }^{1}$ Department of Stomatology, General Hospital of Shenyang Military Area Command, Shenyang 110840, Liaoning Province, P.R. China ${ }^{2}$ Department of Biophysics, College of Basic Medical Science, China Medical University, Shenyang 110001, Liaoning Province, P.R. China

*Corresponding author: Tel./Fax: +86 24 83978251; E-mail: zyxdzhang@163.com

Key Words: W/O, Microemulsion-sol-hydrothermal method, Microtube capsule.

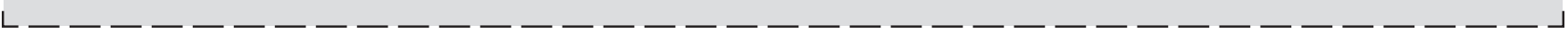

\section{INTRODUCTION}

Capsule has received much attention in recent years due to its controllability in shape and size. Capsule is a kind of metallic, metalloid and organic enclave with a core-shell structure $^{1}$. The micro/nano capsule, with the dimension scale from several nanometers to tens of micrometers, can encapsulate tiny drug molecules. In this way, the activity of the drugs can be enhanced and the rate of drug release is controllable by adjusting the dimension of the drugs and micro pore ${ }^{2}$. Furthermore, the micro/nano capsule for drugs is promising in drug targeting and controlled release owing to the versatility of shell materials and easily crossing blood brain barrier. Therefore, the investigation of micro/nano capsule as a kind of carrier for drug delivery system is a hot issue both in the field of material science and medical science.

Organic polymer used to be adopted as carriers in drug delivery system ${ }^{3,4}$. However, for most organic polymer, the mechanical strength, chemical stability and biocompatibility is poor, especially, some of polymer is toxic, which hindered the application of polymer shell in vivo. In contrast, inorganic material especially inorganic ceramic is considered as a promising candidate for medical capsule shell owing to its excellent mechanical and biological properties ${ }^{5}$. Silica and zinc oxide inorganic ceramics are considered as desirable capsule shells owing to their favourable mechanical stability, chemical stability and biocompatibility ${ }^{6-12}$. In our previous study, silica hollow capsules were prepared via water-in-oil (w/o) emulsion method $^{13}$. However, shapes of the capsules are difficult to control owing to the instability of emulsion. In contrast, $\mathrm{ZnO}$ is versatile in shape controllability ${ }^{14}$, making it superior in preparation of capsule shells. To date, $\mathrm{ZnO}$ hollow capsules has been prepared by various wet-chemical methods such as sol-gel, microemulsion and hydrothermal method ${ }^{15}$. For solgel method and microemulsion method, the shape and size of hollow capsule is difficult to control rigorously owing to the relative high sintering temperature. For hydrothermal method, reacting solution is sealed in a high pressure reaction kettle and the shape and size of capsules could be controlled owing to the relative low reaction temperature. In this case, the stability of reacting solution plays an important role in preparing the hollow capsule.

In this paper, mixture of w/o emulsion and $\mathrm{Zn}(\mathrm{OH})_{2}$ sol was adopted as reacting solution of hydrothermal method and a microemulsion-sol-hydrothermal method was devised to prepare the $\mathrm{ZnO}$ hollow capsule. Furthermore, the formation mechanism of the system is investigated, which pave the way for the application of $\mathrm{ZnO}$ as medical capsule shell.

\section{EXPERIMENTAL}

All of the reagent used in the experiment are chemical pure. A microemulsion-sol-hydrothermal method was adopted to prepare the $\mathrm{ZnO}$ powder. 
$0.9773 \mathrm{~g}$ of monoethanolamine (MEA) was dissolved in $20 \mathrm{~mL}$ ethanol, then $3.112 \mathrm{~g} \mathrm{Zn}\left(\mathrm{CH}_{3} \mathrm{COO}\right)_{2} \cdot 2 \mathrm{H}_{2} \mathrm{O}$ was added into the solution in the molar ratio of $\mathrm{Zn}^{2+}: \mathrm{MEA}=1: 1$. A transparent sol can be formed after magnetic stirring for $c a$. $0.5 \mathrm{~h} ; 1 \mathrm{~g} \mathrm{C}_{19} \mathrm{H}_{42} \mathrm{NBr}$ (CTAB) was added into $30 \mathrm{~mL}$ cyclohexane, followed by the dropwise addition of $1.8 \mathrm{~mL} n$-butyl alcohol. Afterwards, $1.8 \mathrm{~mL}$ deionized water was dropped into the solution slowly. After magnetic stirring for a while, a transparent emulsion can be formed; $0.15 \mathrm{~mL} \mathrm{Zn}(\mathrm{OH})_{2}$ sol was dropped into the emulsion slowly and a transparent precursor reacting solution can be obtained after magnetic stirring for $c a .20 \mathrm{~min}$. The precursor reacting solution was dispersed by the ultrasonic for $c a$. $10 \mathrm{~min}$, followed by sealed into a Teflon kettle for hydrothermal treatment. After treated at $120^{\circ} \mathrm{C}$ for $2 \mathrm{~h}$, the product was separated by centrifugation and washed ultrasonically in ethanol and acetone for 3 times, respectively. Then, the product was dried at room temperature to obtain the $\mathrm{ZnO}$ powder. The flow chart of experiment is shown in Fig. 1.

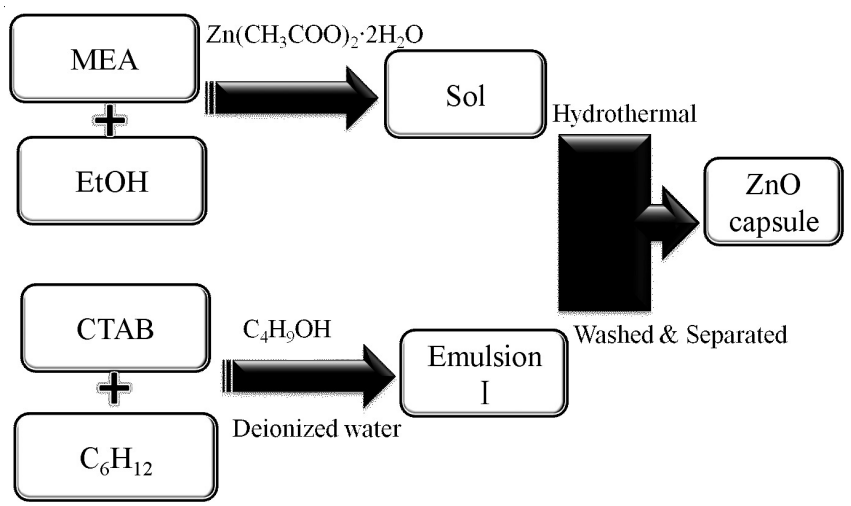

Fig. 1. Flow chart of the experiment

Detection method: SEM (Shimadzu SSX-550) was adopted to observe the morphology of the $\mathrm{ZnO}$ powder; XRD (D/max 3A, Rigaku ) was adopted to investigate the crystallinity of sample.

\section{RESULTS AND DISCUSSION}

Fig. 2 shows XRD pattern of $\mathrm{ZnO}$ powder prepared by the microemulsion-sol-hydrothermal method. A sharp diffraction peak can be found at about $2 \theta=32^{\circ}$, which can be attributed $\mathrm{ZnO}$ crystals according to the standard JCPDS data (No. 36-1451). It is interesting that there is no diffraction peak of other impurities or other orientation of $\mathrm{ZnO}$ crystal, i.e., $\mathrm{ZnO}$ crystal in the experiment has a single orientation. Therefore, it is speculated that $\mathrm{ZnO}$ powder derived from the microemulsion-sol-hydrothermal process has a unique crystal structure

Morphology of powders is shown in Fig. 3. Fig. 3(a) and Fig. 3(b) are the local amplicaiton images. It is shown that $\mathrm{ZnO}$ microtubes can be obtained derived from microemulsionsol-hydrothermal method. From Fig. 3(a), the $\mathrm{ZnO}$ microtubes have a uniform size in length; from Fig. 3(b), the $\mathrm{ZnO}$ microtubes possess a dumbbell structure, the length and diameter of the microtube is about $7 \mu \mathrm{m}$ and $1.5 \mu \mathrm{m}$, respectively. Therefore, the $\mathrm{ZnO}$ sample can be applied as the capsule for drug delivery system and the work of drug loading experiment is in progress.

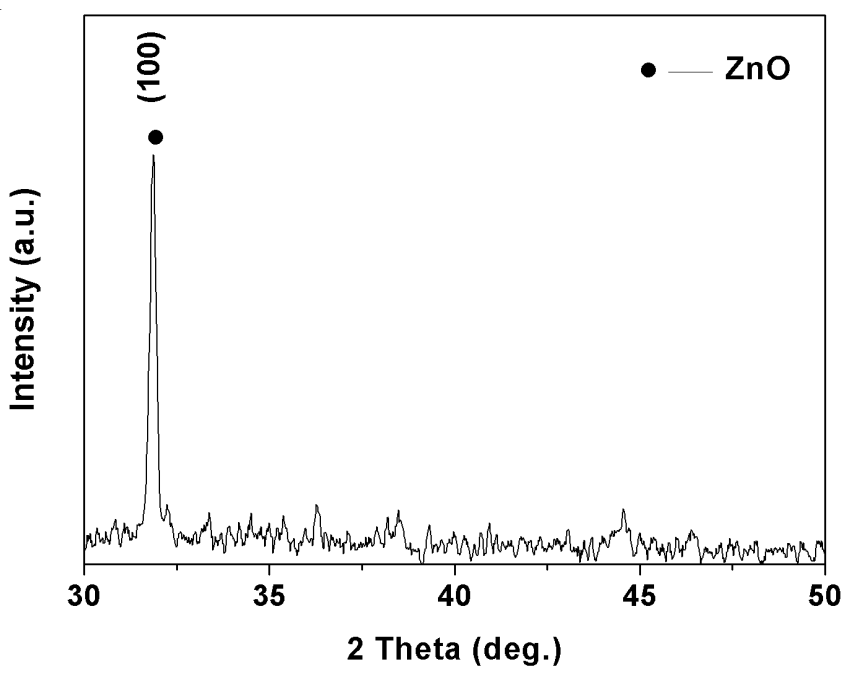

Fig. 2. XRD pattern of $\mathrm{ZnO}$ sample prepared by the microemulsion-solhydrothermal method

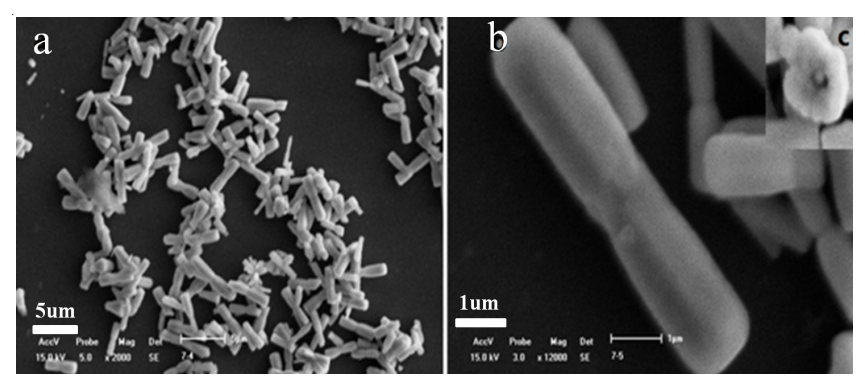

Fig. 3. SEM images of the $\mathrm{ZnO}$ sample. (a) and (b) are the local amplicaiton images

Mechanism model of the reacting solution formation is proposed according to the experimental results (Fig. 4). In the experiment, CTAB and $n$-butyl alcohol is the surfactant and cosurfactant, respectively. At first, monolayer surfactant aggregate at the interface of water and oil (Fig. 4a); when there is other surfactant molecular in the emulsion, hydrophobic group impels the surfactant molecular escape from water phase. As a result, the molecular will auto aggregate to form micelles in the emulsion (Fig. 4b); when the $\mathrm{Zn}$ containing sol was added into the emulsion, the sol tends to aggregate at the interface of micelles due to amphipathy of the sol. As a result, the unique structure of reacting solution is obtained (Fig. 4c). Since monoethanolamine is alkaline, the $\mathrm{Zn}$ containing sol is considered as $\mathrm{Zn}(\mathrm{OH})_{2}$ sol. The formation of the $\mathrm{Zn}(\mathrm{OH})_{2}$ sol can be expressed in eqn. (1). Then, $\mathrm{ZnO}$ crystal can be obtained during the hydrothermal process, which can be expressed in eqn. (2).

$$
\begin{gathered}
\mathrm{Zn}^{2+}+2 \mathrm{OH}^{-} \leftrightarrow \mathrm{Zn}(\mathrm{OH})_{2} \\
\mathrm{Zn}(\mathrm{OH})_{2} \leftrightarrow \mathrm{ZnO}+\mathrm{H}_{2} \mathrm{O}
\end{gathered}
$$

Since the $\mathrm{Zn}(\mathrm{OH})_{2}$ sol tends to aggregate at the interface of micelles, the shape of $\mathrm{ZnO}$ powder is decided by the shape of micelles template. As a result, $\mathrm{ZnO}$ hollow microtube can be obtained, which can be applied as capsules for water-soluble 


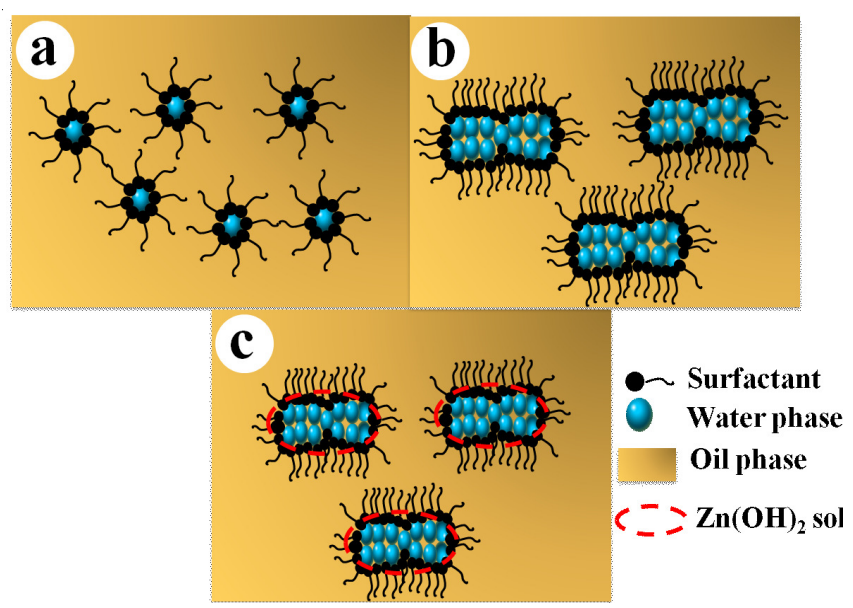

Fig. 4. Schematic formation process for reacting solution

drugs. The relative work on encapsulation of drug is in progress.

\section{Conclusion}

Novel microemulsion-sol-hydrothermal method was adopted to prepare hollow $\mathrm{ZnO}$ hollow microtube as capsule shell. Micelles in the microemulsion are work as template and microscopic shape of the $\mathrm{ZnO}$ capsule is decided by shape of micelles.

\section{ACKNOWLEDGEMENTS}

The author acknowledged the aid and instruction of Prof. Y. Qi, Northeastern University, P.R. China.

\section{REFERENCES}

1. G.R. Duan, A.M. Li, X.J. Yang, L.D. Lu and X. Wang, Micropor. Mesopor. Mater, 116, 86 (2008).

2. W. Zeng, X.F. Qian, Y.B. Zhang, J. Yin and Z.K. Zhu, Mater. Res. Bull., 40, 766 (2005).

3. M.S. Romero-Cano and B. Vincent, J. Control. Release, 82, 127 (2002).

4. M. Changez, K. Burugapalli, V. Koul and V. Choudhary, Biomaterials, 24, 527 (2003).

5. S.-J. Choi, J.-M. OH and J.-H. Choy, J. Ceram. Soc. Jpn., 117, 543 (2009).

6. Z.Z. Li, S.A. Xu, L.X. Wen, F. Liu, A.Q. Liu, Q. Wang, H.Y. Sun, W. Yu and J.F. Chen, J. Control. Release, 111, 81 (2006).

7. J.F. Chen, H.M. Ding, J.X. Wang and L. Shao, Biomaterials, 25, 723 (2004).

8. C. Yagüe, M. Moros, V. Grazú, M. Arruebo and J. Santanaria, Chem. Eng. J., 137, 45 (2008)

9. Y.F. Zhu, J.L. Shi, W.H. Shen, X.P. Dong, J.W. Feng, M.L. Ruan and Y.S. Li, Angew. Chem. Int. Ed., 44, 5083 (2005).

10. N.J. Einerson, K.R. Stevens and W.J. Kao, Biomaterials, 24, 509 (2003).

11. Q. Yuan, S. Hein and R.D.K. Misra, Acta Biomater, 6, 2732 (2010).

12. F. Muhammad, M.Y. Guo, Y.J. Guo, W.X. Qi, F.Y. Qu, F.X. Sun, H.J. Zhao and H.J. Zhu, J. Mater. Chem., 21, 13406 (2011).

13. H.Z. Yang, Y. Ji, X.M. Yu, H. Zhu and L. Zhou, Asian J. Chem., 24, 3507 (2012).

14. C.T. Lee, Materials, 3, 2218 (2010)

15. Q.P. Luo, B.X. Lei, X.Y. Yu, D.B. Kuang and C.Y. Su, J. Mater. Chem., 21, 8709 (2011). 